December 1988

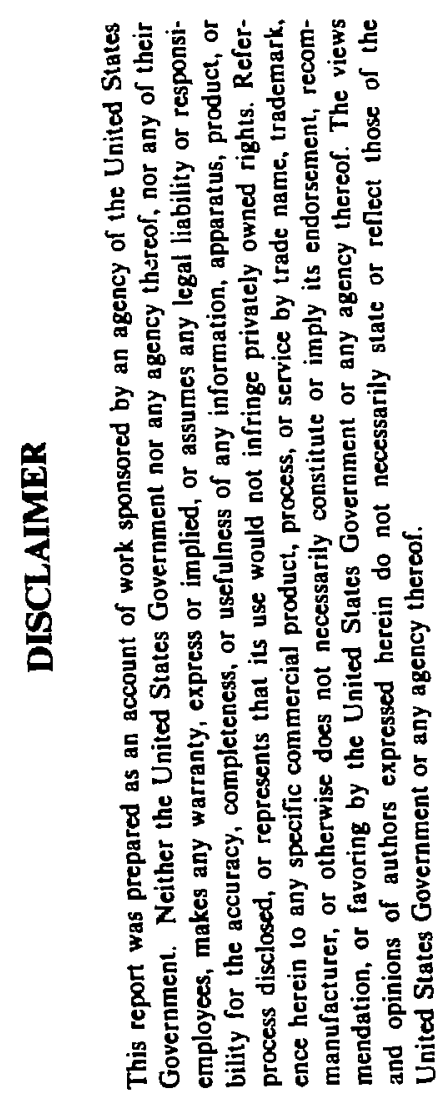

*This work was supported by the Director, Office of Energy Research, Office of Basic Energy Sciences, Advanced Energy Projects Division, U.S. Dept. of Energy, under Contract No. DEAC03-76SF00098. 


\section{HIF/HIFAR Accomplishments: A Synopsis (1)ecember 1988)}

The goal of the HIFAR program is to build a physics and cechnology data base for highcurrent induction linacs using multiple ion beams and current amplification. A central issute is maintaining a low enough 6-D emittance to allow focussing 10 a small focal spot. Acoomplishments include the following:

1. High-current sources of heavy ions have more than adequate brightness:

A suitable injector for a driver should deliver a beam pulse of several amperes at a few $\mathrm{MeV}$. At a time when we imagined a driver to use just one (big) beam starting with a few MeV at a few amperes, we built a 1 -ampere, $1-M V$ cesium injector and determined that its emittance was 100 times smaller than needed in a driver. The surface-ionization source is $30 \mathrm{~cm}$ in diameter and is mounted on the high-voltage terminal of a Marx generator. The beam was further accelerated through three pulsed drift tubes to $2 \mathrm{MeV}$.

\section{Fig. 1: One-ampere cesium source}

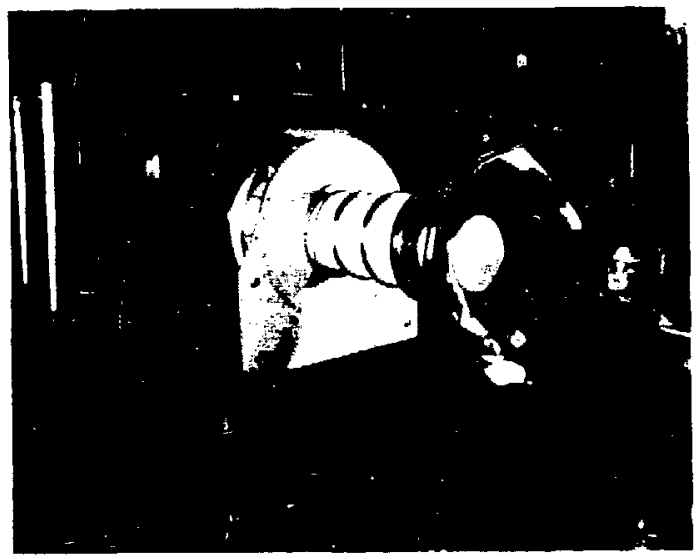

Св) $788-10358$

2. The Single-Beam Transport Experiment established linac transport limits:

Of key importance to driver design is understanding the beam space-charge limit in a strong-focusing linac. In a synchrotron, the Laslett tune-shift criterion allows space-charge defocussing to depress the betatron frequency, $\omega$, by less than $10 \%$ of its low-current value, $\omega_{\mathrm{o}}$. Some years into the HIF studies, the SingleBeam Experiment established in the laboratory that in a long AG transport system (87 electrostatic quadrupoles) very much larger space-charge effects can be tolerated, with the betatron frequency reduced by a factor of 10 from $\omega_{0}$. Down to this value both emittance and current are unaffected provided the betatron phase advance per lattice period is kept below $90^{\circ}$. Simulation shows that below this value image effects may be damaging if the beam is not centered properly.

These studies, which allowed us to consider drivers with ion charge-state $>1$, opened up the field of "space-charge dominated beams" in which the Debye length is a small fraction of the beam diameter.

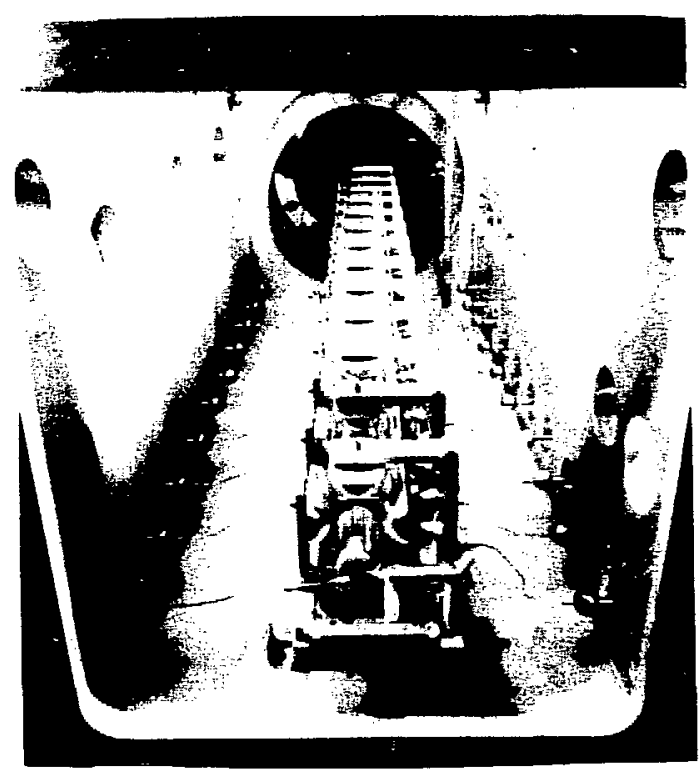

(B) $831-845$

Fig. 2: Two-inch bore quadrupoles in SBTE 


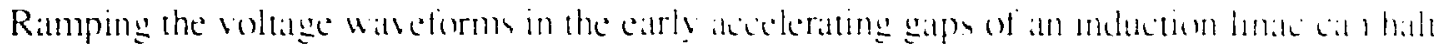



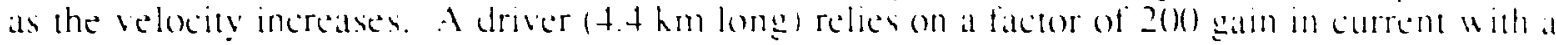


$M V$ through 24 gaps which have shaped woltage pulses applied. Current amplification from 40 mA to $360 \mathrm{~mA}$ has been observed in this $15-\mathrm{m}$ long experiment, although presenty we are studying a less aggressive example (current amplification = 3). Four independenty focusted beams thread the same atcelerating structure; all are space-charge dominated.


XB6 $8810-9680$



(CPP $874-7734$

Fig. 3 (left): Current amplification $(\times 3)$ in MBE-4

Fig. 4 (above): Four-beam ion linac, MBE-4

4. We are developing a high-voltage injector for 16 beams:

One reference driver example calls for 64 beams from a 3-MV injector. As a step in that direction, we are developing a 2-MV, 16-beam injector powered by a gas-insulated $\mathrm{LC}$ generator with a very flat voltage pulse $(<0.1 \%$ for several microseconds). The initial goal is to generate 0.5 amperes/beam of carbon ions (total current $=8 \mathrm{amps}$ ).

Fig. 5: Part of gas-insulated 2-MV injector

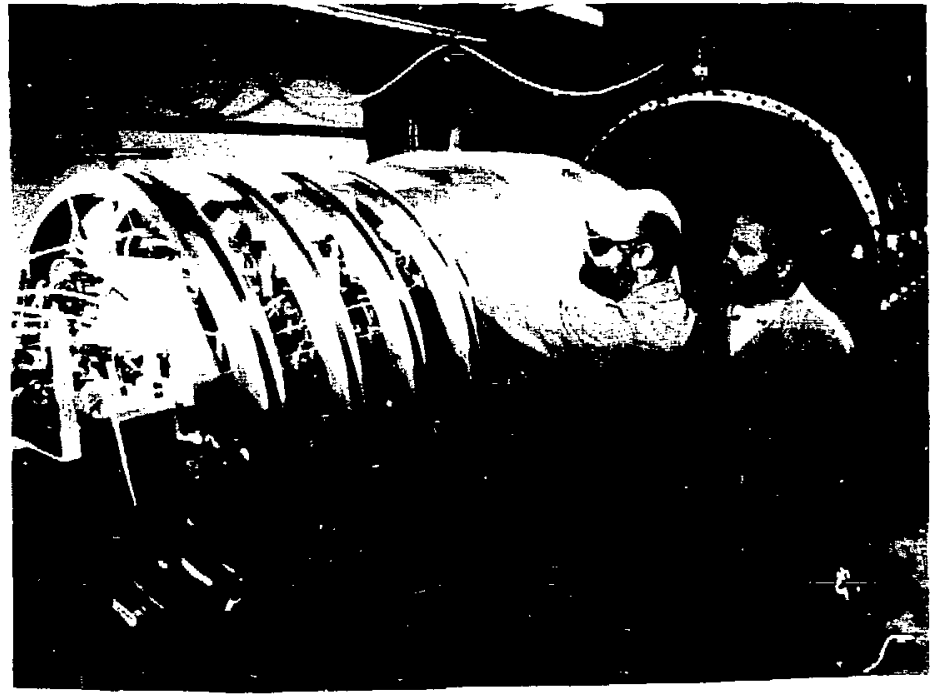

(itis $386-6385$ 
Actually a ciascaded set of six experiments, ILSE is designed to test. in a scaled way with space-chargedominated beams, several important features and beam manipulations to be encountered in a driver. In many key parameters this is a one-tenth scale test. Electric-focussed acceleration of 16 beams is a scale-up from MBE-4. Combining these beams achromatically in sets of four to form four bigger beams involves new beam physics (collectively induced emittance growth). Magnetic-focussed acceleration can be studied for the first time; a light ion, carbon, was chosen to allow convenient magnetic elements at low kinetic energy. One of the emerging $10-\mathrm{MeV}$ beams is selected to study space-charge effects in a largeangle bend, after which it is allowed to bunch in a drift-compression section. In this section, one relies on the collective self-fields at the beam head and tail to remove the velocity shear along the bunch thus avoiding chromatic problems in the final focus. The final focus arrangements allows a variety of experiments on beam propagation, gas and neutralization effects, two-stream instability, etc. A conceptual engineering design for ILSE has been completed and favorably reviewed; the program of experiments would take five years at an enhanced program level of about $13 \mathrm{M} \$ /$ years.

Beyond ILSE, several steps of increasing scale are desirable along the road to a power plant driver. Experiments at $10 \mathrm{~kJ}$, for example, can provide high target temperatures $(>100 \mathrm{eV}$ ), and allow new studies of final focussing as well as the control of high-current longitudinal effects in the accelerator.

\section{The Heavy-Ion Fusion Systems Assessment (HIFSA) gave encouraging results:}

DoE laboratories and industry have collaborated in a broad parametric study of a power plant with an induction linac driver (Fusion Technology, February 1988 issue). A surprisingly broad latitude is found in the choice of accelerator parameters, the projected cost of electricity is favorable (5.5 cents $/ \mathrm{kWh}$ ), and plant sizes down to 500 MWe look reasonable. Driver efficiencies typically lie in the $20-40 \%$ range.

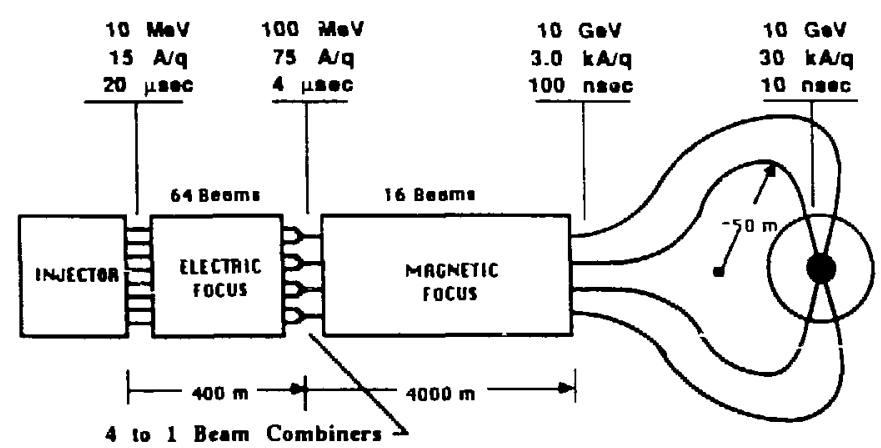

Fig. 7: A cost-effective driver architecture (from HIFSA) 